\title{
ENSENADA EN EL BACHILLERATO: TERRITORIO, POBLACIÓN Y POBLAMIENTO EN EL REINO DE CÓRDOBA A MEDIADOS DEL SETECIENTOS
}

\author{
ENSENADA IN BACCALAUREATE: TERRITORY, POPULATION AND \\ SETTLEMENTS IN THE KINGDOM OF CÓRDOBA IN THE MID-EIGHTEENTH- \\ CENTURY
}

\author{
Mạ Soledad Gómez Navarro \\ hi1gonas@uco.es \\ Facultad de Filosofía y Letras, Universidad de Córdoba (España) \\ Eduardo Lama Romero \\ lamaromero.eduardo@gmail.com \\ IES Ángel de Saavedra, Córdoba (España)
}

Recibido: $13 / 02 / 2020$

Aceptado: 20/06/2020

\begin{abstract}
Resumen:
El presente trabajo es una primera aportación dimanada del Proyecto de Innovación Docente 2018-4-4009, aprobado, con el indicado código, por la Universidad de Córdoba dentro de su programa de Proyectos de Innovación Docente, modalidad 4: Proyectos de Innovación que impulsen la colaboración con centros docentes públicos no universitarios. Titulado "El Catastro de Ensenada en Bachillerato", pretende el estudio de una fuente primaria histórica del siglo XVIII como es el catastro de Ensenada, por parte de estudiantes no universitarios, en el marco de colaboración que aquella modalidad establece; desarrollado en su primera fase durante el curso académico 2018-2019 en el IES "Ángel de Saavedra" de Córdoba por estudiantes de aquel nivel coordinados por quienes suscriben este texto como responsables del citado proyecto, lo que este texto recoge es el análisis de las cuestiones del "Interrogatorio General" del catastro ensenadista más relacionadas con el espacio, moradores y formas de ocupación del espacio.
\end{abstract}

Palabras claves: Innovación Docente, Universidad, Bachillerato, Ensenada, Córdoba, siglo XVIII

\begin{abstract}
:
The present work is a first contribution derived from the Teaching Innovation Project 2018-44009, approved, with the indicated code, by the University of Cordova within its Teaching Innovation Projects program, modality 4: Innovation Projects that promote the collaboration with public non-university teaching centers. Titled "The Cadastre of Ensenada in Bachelor", it intends the study of a historical primary source of the eighteenth century, such as the Ensenada
\end{abstract}


cadastre, by non-university students, within the framework of collaboration established by that modality; developed in its first phase during the academic year 2018-2019 at the IES "Ángel de Saavedra" of Cordova by students of that level coordinated by those who subscribe this text as responsible for the aforementioned project, what this text includes is the analysis of the issues of the "General Interrogation" of the cadastre of the most related to space, inhabitants and forms of occupation of space.

Key Words: Teaching Innovation, University, Bachelor, Ensenada, Cordoba, 18th century

\section{Introducción}

El presente trabajo constituye un primer resultado tangible del Proyecto de Innovación Docente 2018-4-4009, aprobado, con el indicado código, por la Universidad de Córdoba dentro de su programa de Proyectos de Innovación Docente, modalidad 4: Proyectos de Innovación que impulsen la colaboración con centros docentes públicos no universitarios.

Titulado "El Catastro de Ensenada en Bachillerato", pretende el estudio de una fuente primaria histórica como es el catastro de Ensenada, por parte de estudiantes no universitarios, en el marco de colaboración que aquella modalidad establece. Llevado a cabo, en su primera fase -pues se nos ha concedido renovación en la reciente convocatoria de los proyectos de innovación para el próximo curso académico-, durante el curso académico 2018-2019 en el IES "Ángel de Saavedra" de Córdoba por cuarenta y cuatro alumnos y alumnas de 2을 de Bachillerato de Ciencias Sociales y Humanidades, a los que agradecemos muy sinceramente su colaboración porque sin cuya principal, que se reconoce nuevamente, no hubiese sido posible llevarlo a cabo al ser los estudiantes los directos protagonistas de esta experiencia ${ }^{1}$, coordinados por $M^{a}$ Soledad Gómez Navarro, catedrática de Historia Moderna en la Universidad de Córdoba, y por Eduardo Lama Romero, profesor de Educación Secundaria en el referido centro educativo, como responsables del citado proyecto, y con la colaboración de la profesora Ma Teresa Martín Martínez, profesora del mismo indicado IES, presentó un avance de sus resultados en la Jornada de Innovación DIM-EDU, "6o Encuentro de Centros Innovadores en Andalucía", celebrado en la Universidad de Córdoba el dos de octubre de 2019, y a cuyo responsable, el profesor Pere Marquès agradecemos el acogimiento; así como su receptividad para publicar por extenso dicha presentación en la reconocida Revista Didáctica, Innovación y Multimedia (DIM: http://dimglobal.net/revistaDIM38/revistanewescuelasymuseos.htm\#ensenada), dando cuenta, pues, del desarrollo amplio y completo de la actividad realizada, que se ofrece a la academia, en general, e interesados especializados, en particular, con la esperanza de que inspire comparación, contraste y seguimiento, buscando posibles paralelos; en definitiva, de que sirva, fin último de toda labor investigadora no docente o docente.

\footnotetext{
${ }^{1}$ María Carmona Caballero, Ninoska Chacón, Nerea Chaves Equinas, Mạ Carmen Domínguez Villar, Miguel Escobar Cubero, Yasmina Gálvez Chennaoui, Carmen Gómez Luque, Manuel González Jiménez, Manuel Jesús González Sánchez, Desiré Higueras Ramírez, Ángeles Elena Hualoto Quinde, Laura Jiménez Padilla, Álvaro Labrador Escudero, Raquel Lopera Flores, Mạ del Sol Luque Obrero, Ana Belén Luque Villegas, Fernando Manuel Marín Chuecos, Fátima Márquez Rahmani, Alejandro Martín Burgos, Paula Martínez Lozano, Azahara Mạ Martos Barranco, Jael Martos Santiago, Mạ Carmen Molina Mesa, Mạ José Moreno Moreno, Mạ Dolores Muñoz Luján, Almudena Muñoz Villatoro, Carlos Navarro Samaniego, Daniel Povedano Freire, Miguel Pullana Ruiz, Bruno Ríos Moyano, Elizabeth Rojo Casado, María Ruiz Gutiérrez, Alba Ruiz Pullana, Rafaela Serrano Sillero, Jesús Sierra Bello, Minerva Suárez Santos, Juan Torralbo Moya.
} 


\section{Metodología}

Hasta ahora el Proyecto se ha centrado en el análisis de un determinado número de preguntas del "Interrogatorio General" del catastro de Ensenada, aquellas más relacionadas con las materias de Geografía e Historia de Bachillerato, como son las relativas, respectivamente según su orden en aquella fuente, a la denominación del lugar (pregunta número 1), tipo de jurisdicción (pregunta número 2), especies de frutos recolectados en el término (pregunta número 11), número de vecinos (pregunta número 21), existencia o no de hospitales (pregunta número 30 ), número de pobres de solemnidad (pregunta número 36), número de clérigos existentes en la localidad (pregunta número 38), y existencia de conventos y, en su caso, órdenes, sexo y miembros (pregunta número 39). Ahora mismo están en fase de elaboración las preguntas relativas a los aspectos sociales, y ha concluido el estudio de las concernientes a determinados asuntos de la económica agraria -cultivos, producción-, cuyas conclusiones verán la luz próximamente en la Fundación Española de Historia Moderna; y las respectivas a territorio, vecindario y jurisdicción, materia justamente del presente texto. La estrecha relación entre territorio y producción, especialmente en el Antiguo Régimen, justifican la prioridad y paritaria importancia concedida a ambos observatorios, hasta el punto de que prácticamente constituyen unidad.

Y de ahí también que aclaremos que asimismo, junto a las arriba indicadas, contenido específico, insistimos, de esta aportación, en el Interrogatorio General del catastro de Ensenada hay trece preguntas que hacen referencia directa a la producción agrícola: regadíos y secanos (pregunta número 4), calidades de la tierra (pregunta número 5), arboleda (preguntas números 6, 7, 8 y 13), medidas empleadas (preguntas números 9 y 10), especies de frutos (pregunta número 11), cantidad (pregunta número 12), valor de la producción (pregunta número 14) y derechos y cargas (preguntas números 15 y 16). Analizar la producción agrícola sólo a partir de la pregunta número once -"qué especies de frutos se cogen en el término"- resulta indudablemente parcial pero sirve de primera aproximación a un trabajo mucho más complejo que pretendemos elaborar, y desde luego punto de partida para seguir avanzando, especialmente comparando con otras sólidas aportaciones muy conocidas del mismo ámbito cordobés (Bravo Trenas, 1985; Bugella Altamirano, 2006; López Ontiveros, 1990. Naranjo Ramírez, 1991, 1998; Ortigosa Peñas, 1991: 68-69), o no (Camarero Bullón, 1984; Sánchez Salazar, 1988, entre otras); así como para entrever las múltiples posibilidades que se abren a partir de la fuente catastral ensenadista, como indagar la relación entre sociedad rural y economía agraria (Ferreyra, 2014; Martín Martín, 2007), la indudable influencia al respecto de instituciones específicas del abasto en el Antiguo Régimen (López Pérez-Pérez Morote, 2010), o incluso la cultura alimentaria (Leiva Córdoba, 2001; Gómez Navarro-Villegas Becerril, 2015). En todo caso, para el estudio que se presenta sistematizamos la información en varios apartados y en la historiográficamente bien asentada comarcalización del otrora reino de Córdoba (tablas 1-11).

\section{Resultados}

\subsection{Territorio: lugares y espacios}

El reino de Córdoba, en el Catastro de Ensenada, fue considerado como una provincia de primera clase, esto es, con intendente de máximo rango, y por ende, con el máximo salario, teniendo en cuenta que Castilla se dividió en intendencias de primera clase -Burgos, Córdoba, Extremadura, Granada, León, Madrid, Sevilla, Toledo y Valladolid-, de segunda clase -Cuenca, Jaén, La Mancha, Murcia, Salamanca y Segovia-, y de tercera clase -Ávila, Guadalajara, Palencia, Soria, Toro y Zamora (Camarero Bullón, 2002: 154-155)-. Colindaba al norte con las intendencias de Extremadura y La Mancha, al oeste con la de Sevilla, al este con la de Jaén y al sur con la de Granada. Sus límites se correspondían en general con la actual provincia de Córdoba, si bien 
incluía a Chillón -hoy en Ciudad Real- y Peñaflor -actualmente en Sevilla-, en tanto que Belalcázar e Hinojosa pertenecían a la provincia de Extremadura, y Belmez y Villafranca de la Agujas a la de Jaén.

En el reino de Córdoba había cuatro ciudades y setenta y una villas, algunas de ellas despobladas. En total se hicieron setenta y cinco operaciones censales. Dos ciudades eran de realengo, Córdoba y Bujalance, y dos de señorío, Lucena y Montilla, pertenecientes a los estados del duque de Medinaceli. Determinar qué era una ciudad no resulta fácil. En el Antiguo Régimen la "Iuenga y brillante historia" (López Ontiveros, 1990: 14), y en algunos casos el ser cabeza de un reino o la concesión de esta categoría por parte del rey eran motivos relevantes, como lo era su entidad urbana, su población, las dignidades eclesiásticas que albergaba, y sus actividades económicas.

Las villas contaban con sus autoridades, jurisdicción, no siempre unos habitantes, y en algunos casos devengaban rentas en favor de los señores o de la Monarquía. En este sentido Ferrer y González (1996: 26-28) discriminan las tierras de realengo entre aquellas en sentido estricto, en las que la monarquía nombra cargos y cobra rentas en su caso, y las tierras realengas de "señorío terminiego" en que la jurisdicción real, algo impreciso, "formal y honorífico", se limita al nombramiento o sanción de cargos, en tanto que la jurisdicción civil y criminal y las percepciones que genera corresponde a la villa o ciudad. Se daba la existencia de "composiciones polinucleares" (Ferrer y González, 1996; 28), como en Fuente Obejuna, si bien poco frecuentes en el reino de Córdoba, integradas por un núcleo central y varias aldeas. Se incluían los despoblados, a veces con algunas casas abandonadas, centros de grandes cortijos, como realiza López Ontiveros (1990: 5), quien, al analizar los diecinueve despoblados que se citan alrededor de Córdoba, lo hace incluyéndolos en la intervención de Córdoba ciudad. Integran en su conjunto el $12 \%$ del término, consistiendo en "excelentes cortijos". De doce de ellos los nobles son titulares, perteneciendo tres al inmenso patrimonio andaluz del conde de Priego; dos son de instituciones religiosas y cinco de personas sin título; balance que, según el citado investigador, pone sobre la pista de dos ideas fundamentales, a saber: por un lado, la señorialización progresiva del patrimonio regio, muy marcada en la Campiña cordobesa a partir de las ventas efectuadas por Felipe III y Felipe IV; y, por otro, la estructura "de gran propiedad cordobesa del siglo XVIII". En todo caso, esos indicados grandes cortijos son referidos en las operaciones censales de los municipios que los abarcaban o cercanos, como en el caso de la ciudad de Córdoba, y otras veces gozaban del carácter de "villa" o "aldea" pero sin población.

Otro tema importante relacionado con el territorio era el "término". En cada una de las operaciones censales, recogida en la cuestión número 3 , se formulaban una serie de preguntas relacionadas con el término, para delimitar qué territorio comprendía: circunferencia en leguas y tiempo que se tarda en recorrer, longitud de levante a poniente y de norte a sur, linderos por cada uno de los puntos cardinales y figura. La complejidad y la falta de homogeneización propia del Antiguo Régimen son aquí más notorias. Según la Instrucción que acompañaba al Real Decreto de 10 de octubre de 1749 el término era la "unidad territorial base", si bien no se establecía exactamente en qué consistía, como oportunamente se ha señalado: "Aunque la palabra término pueda parecer a veces equívoca, todo parece indicar que en la mayoría de las ocasiones hace referencia al término jurisdiccional", es decir al "territorio jurídicamente encomendado a esa población" (Ferrer Rodríguez, 2002: 401). El dibujo del perímetro del término que se acompañaba y el detalle de sus límites constituyen una valiosa fuente para el estudio del territorio, pero no son precisos ni las medidas "ni el dibujo de su figura" (Ferrer Rodríguez, 2002: 31-32). Más aún, aunque en alguna operación catastral se haga referencia, no 
queda claro qué tipo de leguas se emplean, si bien las leguas de 5.000 varas son lo más frecuente ${ }^{2}$.

A la nobleza, ostentadora de jurisdicción sobre determinadas villas, muchas de ellas despobladas, el Catastro le dio la ocasión de explicitar oficialmente en qué consistía esta jurisdicción, sus límites -no del todo claros en muchos puntos- y el territorio que abarcaba. Particularmente importante es la jurisdicción sobre despoblados -y en la provincia de Córdoba hay bastantes- pues suponía la jurisdicción sobre tierras, que no sobre personas, algo que se revelará en el futuro muy importante, al insinuarse en ello el intento de oficializar esta vinculación, como se ha esgrimido (López Ontiveros, 1990: 15).

La correspondencia entre los términos antiguos y los actuales es muy difícil de establecer. Amparo Ferrer realiza un mapa de jurisdicciones, indicando dónde se han producido los cambios (Ferrer Rodríguez, 2002: 404). La existencia actual de municipios no existentes en el siglo XVIII y la desaparición de otros existentes en el Catastro, dificulta su estudio. La extensión del antiguo reino de Córdoba se cifra en $12.638,21 \mathrm{~km} 2$, prácticamente idéntica a los $12.968 \mathrm{~km} 2$ aportados por Peñín Rodríguez (1991: 115), resultando más fácil determinar la extensión por áreas que por municipios ${ }^{3}$. Para su estudio hemos empleado la comarcalización básica e historiográficamente muy asentada de valle estricto del Guadalquivir, Sierra Morena, Campiña y Subbéticas.

\section{El valle estricto del Guadalquivir}

El valle del Guadalquivir, "valle estricto" lo denominaremos para evitar confusiones, estaría integrado por todas aquellas localidades con límites al gran río. Si contabilizamos sus términos actuales, el territorio abarcaría 3.930,89 km2, el 31,10\% del total ${ }^{4}$, si bien hay que contar los dos términos más grandes de la provincia, Córdoba y Hornachuelos, no estrictamente de valle en ninguno de los dos casos. Así comprende Córdoba, ciudad que se encuentra en una depresión a orillas del Guadalquivir y al pie de Sierra Morena. Tiene un extenso término, que hoy conserva casi igual, modificado por la entrada en el mismo de algunos espacios como Santa Cruz. Igual ocurría con Montoro. Emplazada sobre un meandro del Guadalquivir, la mayor parte de su jurisdicción era pura sierra, ya que incluso comprendía el actual término de Cardeña. A efectos de este trabajo se ha considerado el término actual de Montoro como la superficie del valle estricto, en tanto que el término de Cardeña se ha incorporado a Sierra Morena.

En el valle del Guadalquivir, además, se incluían, aguas abajo de Córdoba, las villas de Peñaflor, en el extremo occidental del reino; Palma del Río, en la confluencia del Guadalquivir y el Genil, con un término entre sierra y vega; Hornachuelos, en la falda de Sierra Morena cordobesa, con la mayor parte de su término en la sierra, lindero en el norte con el de Fuente Obejuna. Más al este, Posadas presentaba un término separado en dos por el río ${ }^{5}$, y Almodóvar del Río. Al sur

\footnotetext{
${ }^{2}$ Una vara son 84 centímetros, por lo que 5.000 varas serán, aproximadamente, 4,2 kilómetros. Sin embargo, en este trabajo vamos a usar la legua castellana de 6.666,66 varas, esto es, 5,57 kilómetros aproximadamente.

${ }^{3} \mathrm{~A}$ tal efecto se ha contabilizado la superficie actual de cada uno de los términos actuales y de los nuevos municipios, no existentes en el siglo XVIII. Todas las superficies están extraídas de los datos oficiales del Instituto de Estadística y Cartografía de Andalucía (IECA).

${ }^{4}$ Se ha considerado que el término actual de Cardeña, incluido en el antiguo de Montoro, se incorpora a la zona de Sierra Morena. Estaba despoblado casi en su totalidad.

${ }^{5} \mathrm{~A}$ efectos de este trabajo se ha considerado el término actual de Fuente Palmera; en realidad, se formó con tierras de distintas jurisdicciones.
} 
del Guadalquivir, Guadalcázar compartía también territorio, conectando las villas del valle estricto con las de la campiña. Además en esta zona se hallaban las villas despobladas de Almenara, cercana a Peñaflor, con características de sierra, la de Mingaobes, encajada en meandros del Guadalquivir, Ascalonías, Moratalla y Villaseca.

En el valle estricto del Guadalquivir, aguas arriba de Córdoba ciudad, se encontraban las villas de El Carpio, Pedro Abad, Montoro, con una jurisdicción muy extensa, a ambos lados del río, comprendiendo ricas tierras de cultivo de vega y extensas zonas de sierra, limítrofes con la de Conquista -hoy se ha dividido por la aparición del municipio exclusivamente serrano de Cardeña- y Aldea del Río, actual Villa del Río, a orillas del Guadalquivir. Tres villas despobladas completaban la zona del valle alto del Guadalquivir en la provincia antigua de Córdoba: Villaverde, Vega de Armijo y Ardales, con buenos cortijos, muy próximos al cauce principal. Villafranca de las Agujas, entre Adamuz y El Carpio por el Este y Córdoba por el Oeste, era una interrupción extraña, pues estaba incluida en el reino de Jaén.

\section{Sierra Morena}

Sierra Morena, con una superficie de $5.007,87 \mathrm{~km} 2$, lo que representaba el $39,62 \%$ del territorio ${ }^{6}$, estaba integrada por veinte villas, algunas con mucha personalidad. Tal era el caso de Fuente Obejuna. Antigua Mellaria romana, ocupaba el ángulo Noroeste de la provincia, y era un conjunto formado por un núcleo principal con 467 vecinos y otros 909 repartidos por veintisiete aldeas: Valsequillo, Granjuela, Esparragosa, Los Blázquez, Prados, Cuenca, Coronada, Canalejas, Argallón, Argalloncillo, El Rubio, Piconcillo, Morenos, Cardenchosa, Posadilla, Navalcuerno, Domarcos, Ojuelos Altos, Ojuelos Bajos, Los Pánchez, Alcornocal, Cumbre, Cañada del Gamo, Lobatón, Doña Rama, Hoyo y Peñarroya. Constituía una forma de poblamiento muy peculiar, pues en un áspero territorio la población se agrupaba en pequeñas aldeas, próximas entre sí. En el mismo valle del Guadiato se encontraba Villanueva del Rey, limítrofe con Belmez. Era esta villa y jurisdicción una encomienda vacante de la Orden de Calatrava, vinculada a Martos, en el Santo Reino. También se hallaban las villas de Espiel con su aldea de Villaviciosa, y Villaharta. Hacia el Sur, Santa María de Trassierra, en las proximidades de Córdoba, entraba en contacto con el valle estricto del Guadalquivir. Obejo ocupaba un espacio central en la sierra, entre la parte occidental y la oriental de Sierra Morena cordobesa.

Las Siete Villas de los Pedroches -Alcaracejos, Torremilano, Pedroche, Torrecampo, Pozoblanco, Villanueva de Córdoba y Añora- comprendían un término municipal muy extenso, proindiviso, en esa comarca. Al Norte, Santa Eufemia, El Viso, El Guijo y Villaralto constituían su extremo septentrional y, más al Norte de estas, en los confines de la provincia, se hallaba Chillón. Finalmente, Conquista, al Este, cerraba la Sierra Morena cordobesa, cerca del Guadalmez, en contacto con el reino de Jaén.

\section{La Subbética}

La Subbética cordobesa estaba integrada por una ciudad y nueve villas. Comprendía una superficie aproximada de 1.548,25 Km2, cifra asimismo muy similar a los $1.466,22 \mathrm{~km} 2$

\footnotetext{
${ }^{6}$ Cifra extraída a partir de la superficie de los términos actuales. Como ya se ha indicado, está incluido el término de Cardeña, en el siglo XVIII incluido en Montoro.
} 
aportados por Peñín Rodríguez (1991: 135$)^{7}$. Ello representaba el 12,25\% del total de la superficie del antiguo reino. Lucena era un importante núcleo urbano, comprendiendo las aldeas de Encinas Reales y Jauja. La villa de Cabra se encontraba al pie de las Sierras Subbéticas. En sus estribaciones, limítrofes con la Alta Campiña cordobesa, se encontraban Luque, Zuheros y Doña Mencía. A mayor altitud se hallaban Priego de Córdoba, Carcabuey y Rute. Finalmente, en el valle del Genil, los términos de Benamejí, con la aldea de Palenciana, e Iznájar cerraban por el sur el reino de Córdoba.

\section{La Campiña}

La Campiña cordobesa estaba integrada por dos ciudades, catorce villas y algunos despoblados sobre un territorio de $2.151,18 \mathrm{~km} 2$ (17\% del territorio). La ciudad de Montilla articulaba la Campiña occidental cordobesa, en un paisaje de suaves lomas y vallonadas, entre el Guadajoz y el Genil. Allí se cita el término indiviso formado por el de la propia Montilla, Aguilar, Monturque, Montalbán y, más al Oeste, sobre el Genil, Puente Don Gonzalo -actual Puente Genil-. Junto a este se hallaban La Rambla, Fernán Núñez, Montemayor, San Sebastián de los Ballesteros y Santaella, con las aldeas de la Guijarrosa y las Huertas de la Boca del Soldado-, además de algunos despoblados, como la villa de Cuartos de Pedro Carrillo, muy próxima a Fernán Núñez, y hoy desaparecida, y las de Maestre Escuela, Santa Cruz de los Llanos y Matachel ${ }^{8}$, relacionados con La Rambla, completaban esta parte de la Campiña Occidental, zona muy fértil, fuertemente agrarizada, señorializada y muy poblada.

El valle del Guadajoz era la divisoria entre la Campiña Occidental y la Oriental. De Sur a Norte se encontraba el despoblado de Silera de Albendín, la villa de Baena y la de Valenzuela. Castro del Río, Espejo, y Santa Cruz. La parte oriental de la Campiña cordobesa estaba ocupada por una ciudad, Bujalance, y las villas de Cañete y Morente, cuyos términos municipales entraban en contacto con los de Córdoba y las villas del valle estricto del Guadalquivir.

\footnotetext{
${ }^{7}$ Estos datos están extraídos, como los anteriores, de las estadísticas oficiales del IECA. En esta estadística se incluyen las superficies de los términos de Almedinilla y Fuente Tójar, insertos en la antigua jurisdicción de Priego.

8 Las operaciones catastrales no se atienen a ningún criterio con respecto a los despoblados. Hay despoblados que se incluyen en las de una ciudad o villa, como los diecinueve despoblados del caso de Córdoba, y en otros casos, como estos, son explícitas. Era muy importante para los miembros de la nobleza significar el grado de dominio sobre determinadas tierras extensas y ricas.
} 
llustración 1: El antiguo reino de Córdoba:

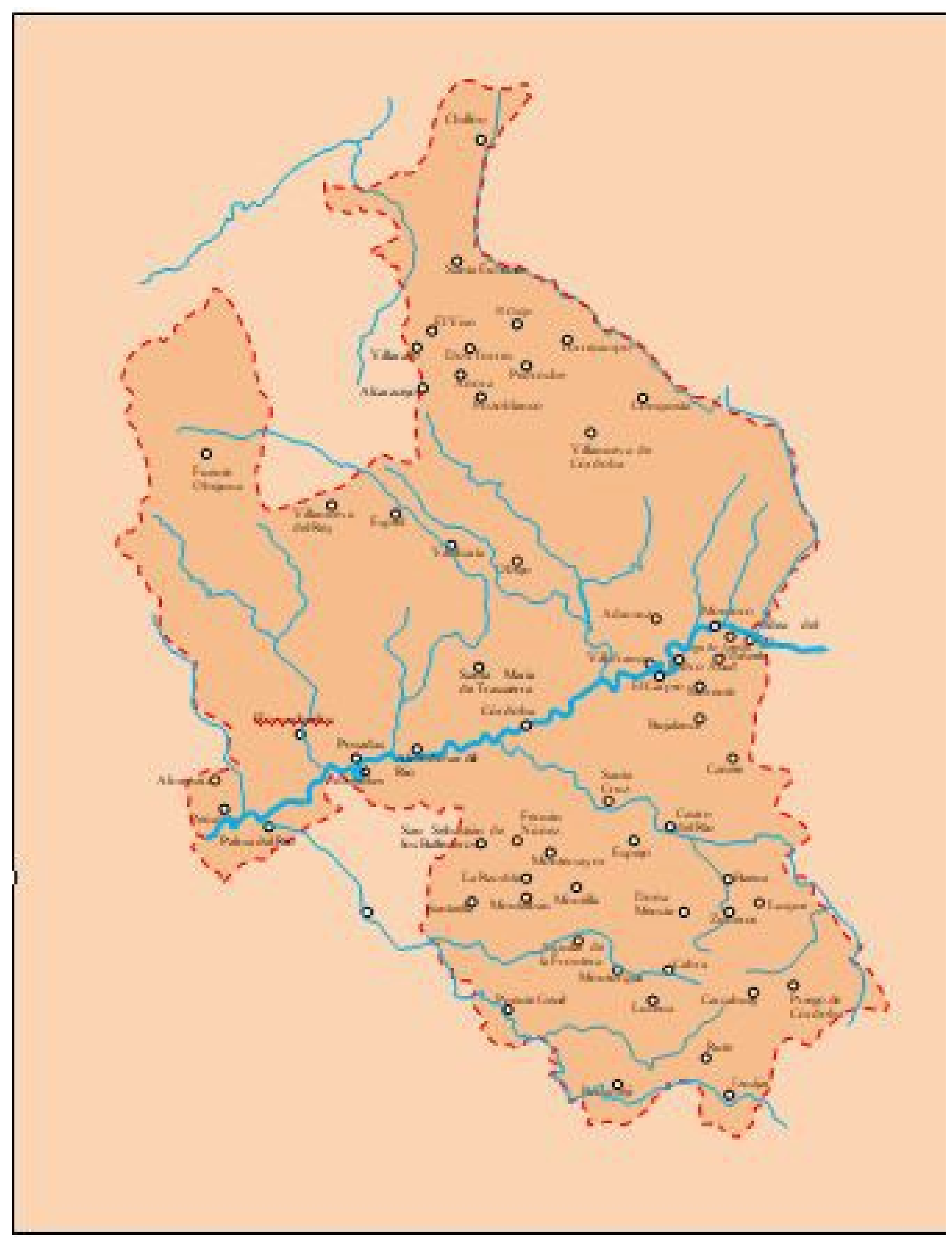

Fuente: Elaboración propia a partir de la información ad hoc del Interrogatorio General 


\subsection{Demografía: población y poblamiento}

El estudio de la población en el Catastro de Ensenada choca en principio con varias dificultades: los datos son pocos fiables en algunos casos, no se atienen a criterios uniformes de medida de población y están expresados en vecinos.

\section{Los vecinos}

Determinar la población exacta que existía en cada una de las localidades en 1752 resulta muy complicado, pese al afán de uniformización de la información que se busca en el Catastro. Para ello hemos de irnos a las respuestas que se dan a la cuestión número 21: "¿De qué número de vecinos se compone la población y cuántos en las casas de campo o alquerías?".

En principio son dos las informaciones que se pueden extraer: el número de vecinos (dato de población) y el número de vecinos dispersos en la jurisdicción (dato de poblamiento). Una respuesta, recogida al azar, la de la villa de Cabra, nos muestra un modelo de respuesta a esta doble cuestión:

"El número de vecinos de que se compone esta población es de presente dos mil doscientos y once, y de ellos los cuarenta y dos son eclesiásticos que hacen cabeza de casa, presbíteros y capellanes, de suerte que quedan reducidos los seglares a dos mil ciento sesenta y nueve vecinos, y de estos viven en las alquerías o casas de campo que hay en el término de esta villa veinte y nueve" ${ }^{\prime 9}$.

La primera cuestión que surge es qué es un vecino. No es un habitante, sino una unidad familiar nuclear castellana, compuesta por todas las personas "que viven en el hogar paterno", por lo que no contempla los independizados -matrimonio, trabajo, emigración, otras situaciones- ni nos da información sobre natalidad, mortalidad o número de hijos por matrimonio (Peñín Rodríguez, 1991: 51). Una comprobación de la veracidad de estos datos se puede obtener a través del análisis de los Libros de Familias de Seglares. Como indica Pilar Peñín (1991: 117), los datos son bastante fiables, pues los diez municipios de las Subbéticas tienen 14.384 vecinos en los Interrogatorios, frente a los 14.340 que refleja la suma de los contenidos en los Libros de Familias de Seglares. En el caso de Córdoba ciudad, los datos son muy aproximados. Concuerdan sólo parcialmente con otras fuentes recogidas por Antonio López Ontiveros (1990: 135): los vecindarios realizados en 1749 (11.979 vecinos) y 1785 (11.534 vecinos), frente a la que se cita en el catastro ensenadista de 10.000 vecinos.

\section{El coeficiente}

Naturalmente, la cuestión importante es traducir los vecinos a habitantes. Ello nos lleva a la cuestión del coeficiente. ¿Qué número de habitantes hay que asignarle a cada vecino? ¿Es igual en todas las localidades? ¿Se puede establecer una media? Además, existe un poderoso elemento distorsionador, que ejemplifica de forma paradigmática Cabra, aunque no siempre la fuente es tan explícita: el número de vecinos comprende a los eclesiásticos que habitan la villa y que se consideran cabezas de casa, y que están contabilizados, a diferencia de los vecinos, como personas físicas individuales. El hecho de que estos eclesiásticos -presbíteros y capellanes- con toda seguridad no viviesen solos, sino acompañados de algún familiar o servidumbre, complica aún más el asunto.

\footnotetext{
9 Respuesta a la pregunta número 21 del Interrogatorio General de la villa de Cabra, con fecha de certificación 1 de julio de 1751 -Libro de Cabra, fo 72v.-.
} 
La unidad familiar la componía "uno o dos hijos, el cónyuge y algún otro familiar o sirviente como mucho [...]. La estimación de cinco contribuyentes sería a todas luces exagerada" (Peñín Rodríguez, 1991: 135). Habitualmente se ha aplicado un coeficiente de 4,5 para el Antiguo Régimen. Cómo determinar el número de vecinos, en el caso de Cabra, se haría de la siguiente forma:

Tabla 1: Población absoluta estimada en Cabra con coeficiente 4,5:

\begin{tabular}{|l|c|c|c|}
\hline Unidades & Vecinos & Coeficiente & Número total de habitantes \\
\hline Vecinos (incluyendo poblamiento disperso) & 2.169 & 4,5 & 9.760 \\
\hline Eclesiásticos cabeza de casa & 42 & & 150 \\
\hline $\begin{array}{l}\text { Clegular que habita en los } \\
\text { conventos (masculinos y femeninos) }\end{array}$ & & & \\
\hline TOTAL & & & 9.952 \\
\hline
\end{tabular}

Fuente: Elaboración propia a partir de la información ad hoc del Interrogatorio General

Mediante el cruce de los datos del Interrogatorio General y de los Libros de Familia, para cada localidad de la Subbética, Pilar Peñín ha calculado el coeficiente que le correspondería (1991: 134): 3,97 en el caso de Iznájar, 3,92 en el caso de Priego, por ejemplo, y así sostiene una media ponderada para la Subbética de 3,87 habitantes por vecino; cifra, por cierto, muy similar a la de obtenida para otra localidad señera del reino de Córdoba en la zona del valle del Guadalquivir como es Palma del Río (Gómez Navarro, 2013: 565). A Córdoba ciudad se le ha aplicado un coeficiente de 3,41 habitantes por vecino -el número de eclesiásticos es mayor (López Ontiveros, 1990: 20)-, cifra que también emplea Matilde Bugella en su trabajo sobre Montilla (2006: 30). Ante la necesidad de homogeneizar el coeficiente para toda la provincia, se ha optado por una cifra intermedia, de 3,75 habitantes por vecino.

\section{La población absoluta}

La población absoluta del siglo XVIII en la provincia de Córdoba sólo se puede obtener por aproximación. Los siguientes cuadros muestran la propuesta que formulamos: 
Tabla 2: Población absoluta del estricto valle del Guadalquivir con coeficiente 3,75:

\begin{tabular}{|c|c|c|c|c|}
\hline Localidades & $\begin{array}{l}\text { Población } \\
\text { Catastro } \\
\text { (vecinos) }\end{array}$ & $\begin{array}{l}\text { Habitantes } \\
\text { estimación (clero } \\
\text { secular incluido) } \\
\text { Coeficiente } 3,75\end{array}$ & $\begin{array}{l}\text { Religiosos } \\
\text { regulares } \\
\text { (monasterios y } \\
\text { conventos) }\end{array}$ & $\begin{array}{l}\text { Población absoluta } \\
\text { estimada }\end{array}$ \\
\hline Peñaflor & 300 & 1.125 & 34 & 1.159 \\
\hline Palma del Río & 1.100 & 4.125 & 128 & 4.253 \\
\hline Hornachuelos & 194 & 727 & Sin datos & 727 \\
\hline Posadas & 500 & 1.875 & 24 & 1.899 \\
\hline Almodóvar del Río & 190 & 713 & 0 & 713 \\
\hline Guadalcázar & 120 & 450 & 20 & 470 \\
\hline Córdoba & 10.000 & 37.500 & 1.642 & 39.142 \\
\hline El Carpio & 464 & 1.740 & 0 & 1.740 \\
\hline Adamuz & 372 & 1.395 & 46 & 1.441 \\
\hline Pedro Abad & 248 & 930 & 0 & 930 \\
\hline Montoro & 1.580 & 5.925 & 20 & 5.945 \\
\hline Aldea del Río & 366 & 1.372 & 0 & 1.372 \\
\hline TOTAL & 15.434 & 57.877 & 1.914 & 59.791 \\
\hline
\end{tabular}

Fuente: Elaboración propia a partir de la información ad hoc del Interrogatorio General 
Tabla 3: Población absoluta de Sierra Morena con coeficiente 3,75:

\begin{tabular}{|c|c|c|c|c|}
\hline Localidades & $\begin{array}{l}\text { Población } \\
\text { Catastro } \\
\text { (vecinos) }\end{array}$ & $\begin{array}{l}\text { Habitantes } \\
\text { estimación (clero } \\
\text { secular incluido) } \\
\text { Coeficiente } 3,75\end{array}$ & $\begin{array}{l}\text { Religiosos } \\
\text { regulares } \\
\text { (monasterios } \\
\text { y conventos) }\end{array}$ & $\begin{array}{l}\text { Población absoluta } \\
\text { estimada }\end{array}$ \\
\hline Fuente Obejuna & 1.376 & 5.160 & 106 & 5.266 \\
\hline Villanueva del Rey & 214 & 802 & 0 & 802 \\
\hline Espiel & 444 & 1.665 & 0 & 1.665 \\
\hline Villaharta & 24 & 90 & 0 & 90 \\
\hline Sta. María de Trassierra & 15 & 56 & 0 & 56 \\
\hline Obejo & 164 & 615 & 0 & 615 \\
\hline Alcaracejos & 212 & 795 & 0 & 795 \\
\hline Pedroche & 450 & 1.687 & 101 & 1.788 \\
\hline Torremilano & 710 & 2.665 & 0 & 2.665 \\
\hline Torrefranca & 163 & 611 & 0 & 611 \\
\hline Pozoblanco & 1.250 & 4.687 & 0 & 4.687 \\
\hline Villanueva de Córdoba & 1.220 & 4.575 & 0 & 4.575 \\
\hline Añora & 260 & 975 & 0 & 975 \\
\hline Santa Eufemia & 254 & 952 & 48 & 1.000 \\
\hline Torrecampo & 480 & 1.800 & 0 & 1.800 \\
\hline Chillón & 554 & 2.077 & 64 & 2.141 \\
\hline El Viso & 370 & 1.387 & 0 & 1.387 \\
\hline El Guijo & 90 & 337 & 0 & 337 \\
\hline Villaralto & 114 & 427 & 0 & 427 \\
\hline Conquista & 43 & 161 & 0 & 161 \\
\hline TOTAL & 8.407 & 31.524 & 319 & 31.843 \\
\hline
\end{tabular}

Fuente: Elaboración propia a partir de la información ad hoc del Interrogatorio General 
Tabla 4: Población absoluta de las Subbéticas con coeficiente 3,75:

\begin{tabular}{|c|c|c|c|c|}
\hline Localidades & $\begin{array}{l}\text { Población } \\
\text { Catastro } \\
\text { (vecinos) }\end{array}$ & $\begin{array}{l}\text { Habitantes estimación } \\
\text { (clero secular incluido) } \\
\text { Coeficiente } 3,75\end{array}$ & $\begin{array}{l}\text { Religiosos } \\
\text { regulares }\end{array}$ & $\begin{array}{l}\text { Población } \\
\text { absoluta } \\
\text { estimada }\end{array}$ \\
\hline Lucena & 4.350 & 16.125 & 341 & 16.466 \\
\hline Cabra & 2.211 & 8.291 & 150 & 8.441 \\
\hline Luque & 734 & 2.752 & 30 & 2.782 \\
\hline Zuheros & 350 & 1.312 & 0 & 1.312 \\
\hline Doña Mencía & 740 & 2.775 & 30 & 2.805 \\
\hline Priego & 2.730 & 10.237 & 128 & 10.365 \\
\hline Carcabuey & 800 & 3.000 & 2 & 3.002 \\
\hline Rute & 1.333 & 4.999 & Sin datos & 4.999 \\
\hline Benamejí & 686 & 2.572 & 17 & 2.589 \\
\hline Iznájar & 300 & 1.125 & 0 & 1.125 \\
\hline Total & 14.234 & 53.190 & 698 & 53.888 \\
\hline
\end{tabular}

Fuente: Elaboración propia a partir de la información ad hoc del Interrogatorio General 
Tabla 5: Población absoluta de la Campiña con coeficiente 3,75:

\begin{tabular}{|c|c|c|c|c|}
\hline Localidades & $\begin{array}{l}\text { Población } \\
\text { Catastro } \\
\text { (vecinos) }\end{array}$ & $\begin{array}{l}\text { Habitantes estimación } \\
\text { (clero secular incluido) } \\
\text { Coeficiente } 3,75\end{array}$ & $\begin{array}{l}\text { Religiosos } \\
\text { regulares }\end{array}$ & $\begin{array}{l}\text { Población } \\
\text { absoluta } \\
\text { estimada }\end{array}$ \\
\hline Montilla & 3.020 & 11.325 & 200 & 11.525 \\
\hline Aguilar de la Frontera & 1.740 & 6.525 & 120 & 6.645 \\
\hline Monturque & 140 & 525 & 0 & 525 \\
\hline Montalbán & 700 & 2.625 & 0 & 2.625 \\
\hline Puente Genil & 1.000 & 3.750 & 80 & 3.830 \\
\hline La Rambla & 1.170 & 4.387 & 105 & 4.492 \\
\hline Fernán Núñez & 1.026 & 3.847 & 0 & 3.847 \\
\hline Montemayor & 511 & 1.916 & 0 & 1.916 \\
\hline San Sebastián Btros. & 1 & 1 & 0 & 1 \\
\hline Santaella & 315 & 1.181 & 0 & 1.181 \\
\hline Valenzuela & 759 & 2.846 & 0 & 2.846 \\
\hline Castro del Río & 1.636 & 6.135 & 81 & 6.216 \\
\hline Espejo & 1.318 & 4.942 & 14 & 4.956 \\
\hline Santa Cruz & 70 & 262 & 0 & 262 \\
\hline Bujalance & 2.148 & 8.055 & 129 & 8.184 \\
\hline Cañete de las Torres & 776 & 2.910 & 22 & 2.932 \\
\hline Morente & 90 & 337 & 0 & 337 \\
\hline TOTAL & 18.420 & 69.072 & 8526 & 69.924 \\
\hline
\end{tabular}

Fuente: Elaboración propia a partir de la información ad hoc del Interrogatorio General

Es necesario aclarar, para esta última tabla, tres precisiones en la columna de religiosos regulares, que, empero, apenas modifican las observaciones que se harán. Dichas puntualizaciones son las siguientes: 1) El dato de los 200 religiosos regulares de Montilla es aproximado, pues el catastro ensenadista solo habla de cinco conventos y dos colegios; teniendo en cuenta que la media de religiosos/as por convento puede acercarse a 40, esa es la cifra propuesta. 2) La cifra de 120 para la de Aguilar de la Frontera es asimismo aproximada, pues sólo se refiere en el Interrogatorio general que hay tres conventos en la villa, aplicándose la misma cifra-promedio que antes. 3) Para Puente Genil, lo mismo, dato aproximado sobre dos conventos. 
En todo caso -y dicho lo cual-, el estudio de la población absoluta del antiguo reino de Córdoba nos apunta alguna certeza y permite formular muchas hipótesis. De acuerdo con nuestro sistema de trabajo -aplicar el coeficiente de 3,75 habitantes por vecino y sumarle el clero regular-, el antiguo reino de Córdoba estaría poblado por 215.446 habitantes. El valle estricto del Guadalquivir, con sus consabidas extensiones sobre Sierra Morena -Montoro, Hornachuelos-, agruparía al 27,75\% de esta población, con el dato destacado de Córdoba capital $(17,40 \%$ del reino), lógico, por otra parte, con su población evaluada en unos 10.000 vecinos aproximadamente, muy apiñada en una ciudad con un casco antiguo muy grande, rodeada por diecinueve despoblados de gran tamaño, como se ha señalado: "El poblamiento cordobés era casi absolutamente concentrado en la capital", dándose un "vacío humano alrededor de la ciudad", por lo demás cultivada en feraces cortijos (López Ontiveros, 1990: 20). Por su lado, la Campiña comprendería el 32,45\%; las Subbéticas, el 25\%, prácticamente la misma cantidad de 26,04\% señalada por Peñín (1991), en definitiva -y en todo caso-, la cuarta parte de la población total; y Sierra Morena, un 14,78\%. Esto significa que había una contraposición entre una parte meridional de la provincia habitada y otra septentrional -sierra Norte sobre todo- despoblada.

En el antiguo reino de Córdoba había siete localidades "grandes", con más de 2.000 vecinos: Córdoba, Lucena, Cabra, Priego, Montilla, Baena y Bujalance. De otra parte, localidades medias, entre 1.999 y 1.000 vecinos, había diez villas: Palma, Montoro, Villanueva de Córdoba, y Pozoblanco, Rute, Aguilar, Puente Genil, La Rambla, Fernán Núñez, Castro y Espejo. Por debajo de 1.000 vecinos destacaba el elevado número de villas de la Sierra-diecisiete- frente al bajo número de la Campiña o del Valle del Guadalquivir -nueve, en cada caso- y de las Subbéticas -solo seis-. Ello nos habla de la distinta forma de ocupación del territorio: los pueblos campiñeses o subbéticos son grandes, muy poblados frente a los de Sierra Morena, pequeñas villas muy diseminadas en el territorio, con poca población y separadas entre sí.

En el catastro ensenadista se consignan trece despoblados, ocho de ellos en el valle estricto del Guadalquivir y cinco en la Campiña. Sin duda se trata de fértiles zonas de cultivo sobre las que determinados nobles querían dejar constancia de su dominio jurisdiccional, aun sin vecinos, para asegurarse la vinculación de ese territorio. Nos confirma lo anterior: la importancia económica de la Campiña y del Valle del Guadalquivir debido a la riqueza de su tierra. Igual ocurría con los diecinueve despoblados que rodeaban Córdoba.

\section{El poblamiento}

De acuerdo con los datos del Interrogatorio General ensenadista, el poblamiento disperso apenas llegaría al $4 \%$ del total de los vecinos del antiguo reino de Córdoba: insignificante en la Campiña $(0,7 \%)$ y el Valle del Guadalquivir $(0,3 \%)$, más importante en las Subbéticas $(14,26 \%)$ y casi inexistente en Sierra Morena, con las excepciones de Fuente Obejuna, con sus 27 aldeas, y Espiel, con un importante núcleo de población en Villaviciosa. Por consiguiente, el poblamiento estaba extraordinariamente concentrado, en villas y ciudades grandes, diseminadas por el territorio, bien delimitadas por murallas, con una separación nítida entre el espacio urbano y el rural ${ }^{10}$. Ello lo confirman diversos estudios, indicándose, en el más centrado específicamente en la ciudad de Córdoba, que su poblamiento estaba "casi absolutamente concentrado en la capital [...] con un vacío humano alrededor" (López Ontiveros, 1990: 21). Quedan por dilucidar los

\footnotetext{
${ }^{10} \mathrm{El}$ recorrido realizado por la Campiña en enero de 2019 con los estudiantes protagonistas del ya indicado Proyecto de Innovación Docente, origen y sustento del presente trabajo, nos permitió realizar observaciones en este sentido, esto es, localidades que han perdido algo de su carácter compacto, y restos de murallas.
} 
factores que han incidido en esta ocupación y las consecuencias que ha tenido sobre el paisaje y la Historia, obviamente cuestiones objeto de otro trabajo.

Tabla 6: Poblamiento del valle del Guadalquivir:

\begin{tabular}{|c|c|c|}
\hline Localidades & $\begin{array}{l}\text { Población } \\
\text { Catastro } \\
\text { (vecinos) }\end{array}$ & $\begin{array}{l}\text { Poblamiento } \\
\text { Catastro } \\
\text { (vecinos) }\end{array}$ \\
\hline Peñaflor & 300 & 0 \\
\hline Almenara & 0 & 0 \\
\hline Palma del Río & 1.100 & 0 \\
\hline Hornachuelos & 194 & 7 \\
\hline Mingaobes & 0 & 0 \\
\hline Ascalonías & 0 & 0 \\
\hline Moratalla & 0 & 0 \\
\hline Villaseca & 0 & 0 \\
\hline Posadas & 500 & 0 \\
\hline Almodóvar del Río & 190 & 0 \\
\hline Guadalcázar & 120 & 22 \\
\hline Córdoba & 10.000 & 0 \\
\hline El Carpio & 464 & 3 \\
\hline Adamuz & 372 & 9 \\
\hline Pedro Abad & 248 & 1 \\
\hline Villaverde & 0 & 0 \\
\hline Vega de Armijo & 0 & 0 \\
\hline Montoro & 1.580 & 8 \\
\hline Aldea del Río & 366 & 0 \\
\hline Ardales del Río & 0 & 0 \\
\hline Total & 15.434 & 50 \\
\hline
\end{tabular}

Fuente: Elaboración propia a partir de la información ad hoc del Interrogatorio General
Tabla 7: Poblamiento de Sierra Morena:

\begin{tabular}{|c|c|c|}
\hline Localidades & $\begin{array}{l}\text { Población } \\
\text { Catastro } \\
\text { (vecinos) }\end{array}$ & $\begin{array}{l}\text { Poblamiento } \\
\text { Catastro } \\
\text { (vecinos) }\end{array}$ \\
\hline Fuente Obejuna & 1.376 & 909 \\
\hline Villanueva del Rey & 214 & 0 \\
\hline Espiel & 444 & 123 \\
\hline Villaharta & 24 & 0 \\
\hline $\begin{array}{l}\text { Sta. } \mathrm{M}^{\mathrm{a}} \text { de } \\
\text { Trassierra }\end{array}$ & 15 & 3 \\
\hline Obejo & 164 & 0 \\
\hline Alcaracejos & 212 & 0 \\
\hline Pedroche & 450 & 0 \\
\hline Torremilano & 710 & 0 \\
\hline Torrefranca & 163 & 3 \\
\hline Pozoblanco & 1.250 & 0 \\
\hline $\begin{array}{l}\text { Villanueva de } \\
\text { Córdoba }\end{array}$ & 1.220 & 0 \\
\hline Añora & 260 & 0 \\
\hline Santa Eufemia & 254 & 4 \\
\hline Torrecampo & 480 & 0 \\
\hline Chillón & 554 & 0 \\
\hline El Viso & 370 & 0 \\
\hline El Guijo & 90 & 1 \\
\hline Villaralto & 114 & 0 \\
\hline Conquista & 43 & 0 \\
\hline Total & 8.407 & 1.043 \\
\hline
\end{tabular}

Fuente: Elaboración propia a partir de la información ad hoc del Interrogatorio General 
Tabla 8: Poblamiento de las Subbéticas:

\begin{tabular}{|l|r|r|}
\hline Localidades & $\begin{array}{l}\text { Población } \\
\text { Catastro } \\
\text { (vecinos) }\end{array}$ & $\begin{array}{l}\text { Poblamiento } \\
\text { Catastro } \\
\text { (vecinos) }\end{array}$ \\
\hline Lucena & 4.350 & 740 \\
\hline Cabra & 2.211 & 129 \\
\hline Luque & 734 & 0 \\
\hline Zuheros & 350 & 010 \\
\hline Doña Mencía & 740 & 0 \\
\hline Priego & 8.730 & 218 \\
\hline Carcabuey & 1.333 & 116 \\
\hline Rute & 686 & 200 \\
\hline Benamejí & 300 & 2.023 \\
\hline Iznájar & 14.184 & \\
\hline TOTAL & & \\
\hline
\end{tabular}

Fuente: Elaboración propia a partir de la información ad hoc del Interrogatorio General
Tabla 9: Poblamiento en La Campiña:

\begin{tabular}{|c|c|c|}
\hline Localidades & $\begin{array}{l}\text { Población } \\
\text { Catastro } \\
\text { (vecinos) }\end{array}$ & $\begin{array}{l}\text { Poblamiento } \\
\text { Catastro } \\
\text { (vecinos) }\end{array}$ \\
\hline Montilla & 3.020 & 20 \\
\hline $\begin{array}{l}\text { Aguilar de la } \\
\text { Frontera }\end{array}$ & 1.740 & 150 \\
\hline Monturque & 140 & 10 \\
\hline Montalbán & 700 & 0 \\
\hline Puente Genil & 1.000 & 9 \\
\hline La Rambla & 1.170 & 70 \\
\hline Fernán Núñez & 1.026 & 0 \\
\hline Montemayor & 511 & 15 \\
\hline $\begin{array}{l}\text { San Sebastián de } \\
\text { los Ballesteros }\end{array}$ & 1 & 0 \\
\hline Santaella & 315 & 0 \\
\hline $\begin{array}{l}\text { Cuartos de Pedro } \\
\text { Carrillo }\end{array}$ & 0 & 0 \\
\hline Maestre Escuela & 0 & 0 \\
\hline $\begin{array}{l}\text { Santa Cruz de los } \\
\text { Llanos }\end{array}$ & 0 & 0 \\
\hline Matachel & 0 & 0 \\
\hline Silera de Albendín & 0 & 0 \\
\hline Baena & 2.000 & 28 \\
\hline Valenzuela & 759 & 0 \\
\hline Castro del Río & 1.636 & 6 \\
\hline Espejo & 1.318 & 2 \\
\hline Santa Cruz & 70 & 0 \\
\hline Bujalance & 2.148 & 0 \\
\hline $\begin{array}{l}\text { Cañete de las } \\
\text { Torres }\end{array}$ & 776 & 0 \\
\hline Morente & 90 & 0 \\
\hline TOTAL & 18.420 & 131 \\
\hline
\end{tabular}

Fuente: Elaboración propia a partir de la información ad hoc del Interrogatorio General

\section{Densidad de población}

La determinación de una densidad de población exige el cruce de datos del siglo XVIII y de la actualidad. Los términos jurisdiccionales se han transformado en términos municipales. Hay algunos que coinciden, otros se han separado con la aparición de nuevos municipios o se han agrupado. Para la población absoluta del siglo XVIII se ha recurrido a aplicar a los vecinos el 
coeficiente del 3,75. Para el territorio, se ha considerado el territorio por comarcas, de acuerdo con los datos del Instituto de Estadística y Cartografía de Andalucía (IECA), excepto para Chillón, en que se han considerado los datos del Instituto Geográfico Nacional (IGN).

Tabla 10: Densidad de población por territorios:

\begin{tabular}{|l|c|c|c|}
\hline Comarcas & $\begin{array}{c}\text { Superficie en } \\
\mathbf{k m}^{\mathbf{2}}\end{array}$ & $\begin{array}{c}\text { Población siglo XVIII } \\
\text { (coeficiente 3,75) }\end{array}$ & Densidad de población \\
\hline Valle estricto del Guadalquivir & $3.930,89$ & 57.877 & 14,72 \\
\hline Sierra Morena & $5.007,87$ & 31.524 & 6,30 \\
\hline Subbéticas & $1.548,27$ & 53.190 & 34,35 \\
\hline Campiña & $2.151,18$ & 69.072 & 16,74 \\
\hline Media provincial & $12.638,21$ & 211.663 & 32 \\
\hline
\end{tabular}

Fuente: Elaboración propia a partir de la información ad hoc del Interrogatorio General

Según estos datos, encontramos una parte sur de la provincia muy poblada, afirmándose 19,3 habitantes por km2 como media andaluza (Estepa Giménez, 1992: 109-118), por lo que el valle del Guadalquivir, y la provincia en su conjunto, estarían alrededor de esas cifras, si bien hay un contraste muy marcado entre la Campiña y las Subbéticas, muy pobladas, y la Sierra Morena cordobesa, con una débil densidad. Ello hace de la provincia de Córdoba un ejemplo de territorio contrastado y complementario, con una sierra despoblada, proveedora de agua y recursos naturales, y una campiña rica en mano de obra y producción agrícola.

\section{La urbanización}

Determinar cuándo una población es urbana o rural resulta hoy día incluso muy difícil. Hay quien recurre a criterios poblacionales. En este sentido, para la consideración de núcleo urbano por su número de habitantes, en concreto para las Subbéticas, se dividen sus núcleos de población estableciendo tres categorías, atendiendo, insistimos, sólo a su población, resultando así núcleos grandes, con más de 2.000 vecinos; medios, entre 1.000 y 500 ; y pequeños, con menos de 500; y afirmándose de esta forma que los núcleos grandes suponían el $30 \%$ y acogían al 62,95\% de la población en las Subbéticas (Peñín Rodríguez, 1991: 110). Pero también quien maneja como criterio la actividad laboral dominante en la localidad, esto es, municipios donde hubiera número significativo de población dedicada al sector secundario o terciario, sería un núcleo urbano. De momento, este criterio escapa de nuestro trabajo. Podría considerarse núcleo urbano aquella localidad en que residiera el representante del poder político a nivel local, del poder eclesiástico con máxima autoridad de jurisdicción -corregimientos y Ordinarios, respectivamente-, o del cultural -colegios mayores, por ejemplo-; o el que tuviera un número más amplio de conventos, pero cuánto sería el número mínimo para ello. Parece que el carácter de ciudad debe depender de otros $-y$ aun varios criterios-, pero el número de habitantes es independiente del estatuto de la ciudad, por lo que hay poblaciones que son villas y tienen más habitantes.

En todo caso, teniendo en cuenta solamente el criterio poblacional -esto es, considerar población urbana aquellos núcleos con más de 2.000 vecinos-, en el Valle estricto del Guadalquivir habría una sola ciudad, la propia Córdoba, que acogería al 64,8\%; en las Subbéticas, tres municipios -Lucena, Cabra y Priego-, lo que representa el 65,5\% de la población; y en la Campiña otros tres -Montilla, Baena y Bujalance-, con un $38,9 \%$ de vecinos. De nuevo el 
desequilibrio extremo entre la Sierra Morena, con pequeños núcleos de población, y las Subbéticas, con grandes núcleos, estando la ciudad de Córdoba como una excepción en medio de todo el territorio.

\section{La comparación con el presente}

Cuando estos datos se ponen en relación con el presente cobran un especial sentido. El antiguo reino de Córdoba ya no existe. En su lugar, con otros contornos y otro territorio, se encuentra la provincia de Córdoba. Sin embargo, se pueden establecer algunas comparaciones y sacar algunas conclusiones.

Tabla 11: Población en el territorio del antiguo reino de Córdoba, 2018:

\begin{tabular}{|l|c|c|c|c|}
\hline Unidades & Población absoluta & $\begin{array}{l}\text { Densidad de } \\
\text { población } \\
\text { Habitantes/Km }\end{array}$ & $\begin{array}{l}\text { Porcentaje de } \\
\text { población } \\
\text { absoluta sobre } \\
\text { el total }\end{array}$ & $\begin{array}{l}\text { Porcentaje de } \\
\text { poblamiento } \\
\text { disperso }\end{array}$ \\
\hline $\begin{array}{l}\text { Valle estricto } \\
\text { del Guadalquivir }\end{array}$ & 411.012 & 104,55 & 54,25 & 3,9 \\
\hline Sierra Morena & 57.366 & 11,46 & 7,57 & 3,2 \\
\hline Subbéticas & 118.612 & 76,60 & 15,65 & 7,1 \\
\hline Campiña & 170.631 & 79,32 & 22,53 & 5,9 \\
\hline TOTAL & 757.621 & 59,94 & 100 & 4,8 \\
\hline
\end{tabular}

Fuente: Elaboración propia a partir de estadísticas del IECA

Más de la mitad de la población del territorio del antiguo reino de Córdoba se concentra en el valle estricto del Guadalquivir, pero Córdoba ciudad, restada la población de Santa Cruz, incorporada a la Campiña, y de Santa María de Trassierra, inserta en Sierra Morena, representa por sí sola el $42,86 \%$ del total, lo que distorsiona los datos. Eso quiere decir que el Sur de la provincia antigua de Córdoba agrupa al $38 \%$ del total, frente al poco más del $7 \%$ del Norte. Significa una gran concentración en la ciudad de Córdoba.

La comparativa es muy significativa. La población absoluta ha aumentado en todas las comarcas, con una media de un $258 \%$, pero este aumento ha sido desigual, pues en el Valle estricto del Guadalquivir ha sido del $610,14 \%$, en buena medida debido a la ciudad de Córdoba, cuya población ha aumentado un $766 \%$. Sierra Morena también ha aumentado en un $81,97 \%$, en tanto que las Subbéticas (123\%) y la Campiña (147\%) lo han hecho en un porcentaje muy parecido. Lo que ha cambiado notablemente es su peso en el total del territorio del antiguo reino. Así, Córdoba, que representaba en el siglo XVIII el 17,7\% de la población, acoge en la actualidad a más de 4 de cada 10 habitantes; Sierra Morena ha descendido del 15\% al 7,5\%; las Subbéticas, de la cuarta parte de la población al 15,65\% y y la Campiña, que acogía a la tercera parte de la población, ha pasado al 22,53\%. Es decir, en Córdoba ciudad hay más habitantes que en la Campiña y las Subbéticas juntas. 
A pesar de todo esto hay villas y ciudades que han visto cómo su población absoluta descendía desde el siglo XVIII. Seis de ellas se encuentran en Sierra Morena (Fuente Obejuna, Pedroche, Dos Torres, Santa Eufemia, Torrecampo y Chillón), dos en las Subbéticas (Zuheros y Carcabuey) y tres en la Campiña oriental (Valenzuela, Espejo y Bujalance). Esto confirma la despoblación de los lugares con mayor altitud o más pequeños. El poblamiento se mantiene muy parecido, pues, frente al $4 \%$ de población dispersa del Catastro, las fuentes del siglo XX arrojan un poblamiento disperso del $4,8 \%$, con notables casos en Fuente Obejuna y Priego.

La tendencia hacia una mayor urbanización queda constatada atendiendo al número de vecinos/habitantes por localidad. En el Catastro de Ensenada hay siete núcleos con más de 2.000 vecinos, en tanto que en 2018 hay 12 municipios con más de 10.000 habitantes, pues se han sumado a Córdoba, Palma del Río y Fuente Palmera (Valle), Pozoblanco (Sierra Morena) y a Montilla y Baena, Aguilar, Puente Genil y la Carlota (Campiña), permaneciendo por encima de los 10.000 habitantes Priego, Lucena y Cabra en las Subbéticas.

\section{Conclusiones}

Al nivel actual del trabajo hasta el presente elaborado, y tras todo lo expuesto, estamos en condiciones de afirmar las siguientes observaciones:

* En la Edad Moderna las unidades territoriales debían contar con un territorio variado y complementario, capaz de proporcionar a sus moradores casi todo lo necesario, dado el alto grado de autoabastecimiento que existía. Un ejemplo a escala era el término de Córdoba, con una parte de sierra, otra de río y otra de campiña. El antiguo reino de Córdoba disponía de este mismo carácter complementario, enriquecido por la presencia de una unidad de relieve diferenciada al sur, como era la Subbética, atravesada por diversos cursos alimentados por los manantiales de las montañas calizas cercanas. Ello proporcionaba, en condiciones normales, la satisfacción interna, casi completa, si bien con sus carencias, de las necesidades fundamentales de la población.

* La ocupación del territorio, de otra parte, era muy diferente en Sierra Morena, con un gran vacío poblacional, que al sur del Guadalquivir, donde los municipios estaban más próximos, en puntos estratégicos, insertos en un territorio con fértiles cortijos muy trabajados.

* La riqueza del territorio en la mitad sur de la provincia se constata por el elevado número de municipios, su carácter señorial y la relación que aflora en toda la información catastral sobre la existencia de grandes cortijos.

* El territorio montañoso del Norte resulta indispensable en esta provincia. Aportaba hombres y mujeres, agua, productos como la madera y espacio.

* Existía, y se mantiene, una gran importancia de la ciudad de Córdoba como lugar de atracción de la población, carácter que se ha reforzado con el paso de los siglos.

* El poblamiento concentrado tiene un dominio casi absoluto en el antiguo reino de Córdoba, y se ha mantenido en nuestros días.

* El catastro de Ensenada pone sobre la pista de la existencia de una gran propiedad, con grandes explotaciones, pero esto deberá ser corroborado por otros estudios. 


\section{Referencias Bibliográficas}

Bravo Trenas, A. (1985). "Propiedad y aprovechamiento de la tierra en Espejo (Córdoba) durante el siglo XVIII". Ifigea: Revista de la Sección de Geografía e Historia, 2, 139-162.

Bugella Altamirano, M. (2006). Montilla según las respuestas generales del Catastro de Ensenada, 1752; Montilla: Asociación de Estudios de Ciencias Sociales y Humanidades.

Camarero Bullón, C. (1984). "La producción agraria en el siglo XVIII". Estudios geográficos, 45 (174), 81-108.

--- (2002). "Vasallos y pueblos castellanos ante una averiguación más allá de lo fiscal: el Catastro de Ensenada, 1749-1756". En I. Durán Boo; C. Camarero Bullón (Coords.). El Catastro de Ensenada: magna averiguación fiscal para alivio de los vasallos y mayor conocimiento de los reinos. Catálogo, (113-388); Madrid: Ministerio de Hacienda.

Estepa Giménez, J. (1992). "Población y sociedad en la provincia de Córdoba a mediados del siglo XVIII". En M. Peláez del Rosal (Coord.). El barroco en Andalucía. Conferencias de los Cursos de Verano de la Universidad de Córdoba sobre "El barroco en Andalucía" (109-118), 2; Córdoba: Universidad.

Ferrer Rodríguez, A. (2002). "La documentación del Catastro de Ensenada y su empleo para la reconstrucción cartográfica". En Ignacio Durán Boo; C. Camarero Bullón (Coords.). El Catastro de Ensenada: magna averiguación fiscal para alivio de los vasallos y mayor conocimiento de los reinos. Catálogo (399-412); Madrid: Ministerio de Hacienda.

Ferreyra, A.I. (2014). "Relaciones sociales en el mundo rural. Córdoba, 1780-1855". Mundo agrario: Revista electrónica de estudios rurales, 15 (30), 1-19.

Ferrer Rodríguez, A.; González Arcas, A. (1996). Las medidas de tierra en Andalucía. Según las Respuestas Generales del Catastro de Ensenada; Madrid: Tabapress.

Gómez Navarro, S. (2013). "Familia, profesión y estado social: la villa de Palma del Río (Córdoba) a mediados del Setecientos", Anuario Jurídico y Económico Escurialense, XLVI, 558-584.

Gómez Navarro, S.; Villegas Becerril, A. (2015). “Entre los pucheros también anda Dios: despensa y (posible) mesa de las carmelitas descalzas a fines del Antiguo Régimen según el convento cordobés de Santa Ana". En Campos y Fernández de Sevilla, J. (Coord.). Santa Teresa y el mundo teresiano del Barroco (93-108); San Lorenzo del Escorial: Real Centro Universitario Escorial-María Cristina.

Leiva Córdoba, F. (2001). Vocabulario cordobés de la alimentación (ss. XV y XVI); Córdoba: Universidad.

López Pérez, M.; Pérez Morote, R. (2010). "La contabilidad de las instituciones municipales del antiguo régimen: el pósito de la ciudad de Albacete". Pecunia: revista de la Facultad de Ciencias Económicas y Empresariales, 11, 177-199.

López Ontiveros, A. (Ed., 1990). Córdoba, 1752: según las respuestas generales del Catastro de Ensenada; Madrid: Tabapress.

Martín Martín, V. O. (2007). "Los regímenes de tenencia indirectos en la agricultura andaluza: evolución y estado actual". Investigaciones geográficas, 43, 19-44.

Naranjo Ramírez, J. (1991). Cultivos, aprovechamientos y sociedad agraria en la campiña de Córdoba: Fernán Núñez y Montemayor (siglos XVIII-XX); Fernán Núñez: AyuntamientoConcejalía de Cultura. 
--- (1998). Génesis del paisaje agrario olivarero-vitícola en la Campiña de Córdoba: Aguilar y Moriles en el siglo XVIII; Córdoba: Diputación Provincial.

Ortigosa Peñas, M. (1991). Propiedad territorial y espacio agrario en Villanueva del Rey; Córdoba: Diputación Provincial-Ayuntamiento de Villanueva del Rey.

Peñín Rodríguez, Mạ P. (1991). La población y poblamiento en la Subbética cordobesa en el siglo XVIII según el Catastro del Marqués de Ensenada; Priego de Córdoba: Ayuntamiento.

Sánchez Salazar, F. (1988). Extensión de cultivos en España en el siglo XVIII; Madrid: Ministerio de Agricultura, Pesca y Alimentación (MAPA). 